\section{Feline bone marrow aspiration: triggers for sampling and disease prevalence}

\section{Alba Maldonado-Moreno ${ }^{1}$, Paola Monti ${ }^{1}$, Paolo Silvestrini ${ }^{2}$, Rachel Miller ${ }^{1}$}

1 Dick White Referrals, Six Mile Bottom, United Kingdom

2 University of Liverpool, Liverpool, United Kingdom

\section{OBJECTIVES}

To determine the clinical settings that lead to bone marrow (BM) aspiration in cats and the prevalence of BM disease according to the initial reason for sampling.

\section{METHODS}

The records of all feline patients undergoing BM examination were retrospectively reviewed. Identified cases were classified into 6 categories based on the reason for the procedure: anaemia, bicytopenia, pancytopenia, neutropenia, tumour staging and other triggers. The final diagnosis was recorded for each patient.

\section{RESULTS}

A total of 93 patients were identified and included in the study. Triggers for BM aspiration included: anaemia (35), bicytopenia (27), pancytopenia (13), neutropenia (8), and tumour staging (7), circulating eosinophilia (1), suspected BM suppression secondary to chemotherapy (1), and thrombocytopenia (1).

No significant BM abnormalities were found in 20 cases from all categories: anaemia (6) group, bicytopenia (4), pancytopenia (1), neutropenia (3), tumour staging (4) and others (2).

Non-regenerative immune-mediated haemolytic anaemia (NR-IMHA) was the most commonly identified disease in the anaemia (23/35), bicytopenia (11/27) and pancytopenia (8/13) groups. In the neutropenic group, immune-mediated neutropenia was most common, diagnosed in 5/8 cats. In the tumour staging group 1 cat had a normal BM, one was diagnosed with multiple myeloma and 1 with neoplastic mast cell infiltration.

\section{STATEMENT (CONCLUSIONS)}

Anaemia and bicytopenia were the most common triggers for BM sampling in cats. Non-regenerative IMHA was the most prevalent disease identified in this study population accounting for $42 / 93$ patients and accounting for $65 \%$ and $40 \%$ of anaemic and bicytopenic cats respectively.

\section{Proteinuria in canine non-erosive immune mediated polyarthritis}

\section{Lucy Barker, Sophie Adamantos, Simon McManus, Vicki Black}

University of Bristol, Bristol, United Kingdom

\section{OBJECTIVES}

To evaluate the point prevalence of proteinuria and assess for association with risk of relapse in dogs with primary non-erosive immune-mediated polyarthritis.

\section{METHODS}

Single-centre retrospective referral-based study. The hospital database was searched for dogs with a diagnosis of primary non-erosive immune-mediated polyarthritis and concurrent urinalysis between January 2009 and August 2018. Data analysed included signalment, investigation findings, treatment and long-term follow-up including repeat UPC results. Dogs were defined as either non-proteinuric (UPC $<0.2$ ), borderline-proteinuric (UPC 0.2-0.5), or overtly-proteinuric (UPC > 0.5). Within the overt proteinuria group those with UPC >2 were defined as markedly-proteinuric. Statistical analysis was undertaken using point prevalence and Chi-squared test to assess presence of proteinuria and whether marked proteinuria was associated with an increased risk of relapse respectively.

\section{RESULTS}

Fifty-eight dogs were eligible for inclusion. Twenty-eight dogs (48\%) were non-proteinuric, 8 dogs (14\%) were borderline proteinuric and 22 dogs (37\%) were overtly proteinuric. Marked proteinuria was present in 12 dogs (20\%). Repeated urinalysis was performed in 9/12 dogs with marked proteinuria, $7 / 9$ (77\%) had improvement to a UPC $<1.2$ (median 0.46, range 0.1-1.2) within 1 month, in four of these dogs improvement was documented despite treatment with prednisolone. There was no association between the presence of marked proteinuria and risk of relapse of clinical signs $(p=0.35)$.

\section{STATEMENT (CONCLUSIONS)}

Proteinuria, including marked proteinuria, was common in this cohort of dogs diagnosed with primary nonerosive immune-mediated polyarthritis, and was not associated with increased risk of relapse of clinical signs. Presence of proteinuria did not appear to preclude prednisolone therapy. 